\title{
Pomegranate juice protects kidneys from Cisplatin-induced nephrotoxicity
}

\author{
Hussain A. Aljebori * \\ Ali H. Abady** \\ Jaffar N. Jaffar Alalsaidissa***
}

\author{
MBChB, MSc, PhD , CABM \\ BSc, MSc \\ MBChB, MSc PhD
}

\begin{abstract}
$\underline{\text { Abstract }}$
Background: Cisplatin is a potent anti-cancer agent used successfully in treatment of cancers of solid organs, but it has a high rate of nephrotoxicity.

JFac Med Baghdad 2017; Vol.59, No .4

Received: Oct. 2017

Accepted: Dec. 2016

Objective: The present study was designed to study Cisplatin-induced nephrotoxicity and the nephroprotective property of pomegranate juice.

Materials and Methods: The experiment was performed on 36 Iraqi white male domestic rabbits. Rabbits were divided into three groups; control group (received neither pomegranate juice, no Cisplatin), Cisplatin group (received Cisplatin only), and pomegranate group (received pomegranate juice and Cisplatin).

Results: Cisplatin group showed marked reduction of renal function manifested by high levels of blood urea, serum creatinine, and low level of serum albumin.Raised levels of oxidative stress markers and severe renal parenchymal damage by histopathology. While, pomegranate group showed almost normal renal function tests and normal levels of oxidative stress markers, and normal renal parenchymal histopathology.

Conclusion: Cisplatin in a highly nephrotoxic drug, and Pomegranate juice has a nephroprotective activity against Cisplatin-induced nephrotoxicity.

Keywords: Cisplatin, nephrotoxicity, Pomegranate.
\end{abstract}

\section{Introduction:}

Nephrotoxicity is an injury resulting in renal damage because of exposure to toxic materials [1]. Although nephrotoxicity is a well-known cause of morbidity and mortality all over the world, its prevalence is increasing even in developed countries $\left[\begin{array}{ll}1, & 2\end{array}\right]$. Nephrotoxic agents are of wide varieties including antibiotics, chemotherapeutic agents, and non-steroidal anti-inflammatory drugs ... etc. [2]. Cisplatin is a potent chemotherapeutic agent, and it is widely used with high cure rate in cancers of ovaries, head and neck, testicular, non-small cell lung cancer, and others [3]. Nephrotoxicity is a major complication of Cisplatin, and clinical studies showed nephrotoxicity in about $1 / 3$ rd of patient using this drug [3]. Since the kidneys are the route of excretion of Cisplatin it is expected to be concentrated in renal tubules especially the proximal renal tubules. Studies showed its concentration in proximal renal tubular epithelium about five times than that of patient serum [4].Cisplatin induces renal tubular and vascular endothelial injury and dysfunction mediated by oxidative stress and inflammation [3, 4]. Several mechanisms were hypothesized to explain the mechanisms of Cisplatin induced nephrotoxicity [5]. Mechanisms of nephrotoxicity and renal injury are

* Dept. of Pathology, College of Medicine, University of Al-mustansiriyah.

**Al-Yarmouk College of Pharmacy.

***Dept. of Pathology,College of Medicine University

of Baghdad, Email: jafaralissa@yahoo.com. many, including induction of apoptosis through DNA damage, and stress of endoplasmic reticulum, inflammatory reaction, oxidative stress with increased levels reactive oxygen species (ROS), reduction of serum and tissues Glutathione GSH level and increased production of tumor necrosis factor- $\alpha$ (TNF$\alpha$ ) in the renal parenchymal cells [5]. The vascular injury induced by Cisplatin may compromise the blood flow inside the kidney resulting in a decrease of glomerular filtration rate, deterioration of renal function and ischemic injury to renal parenchyma [4, 5]. Cisplatin nephrotoxicity is largely the effect of oxidative stress injury initiated by direct action of excess of oxygen reactive species on cell components including lipids in membranes of the cells causing lipid peroxidation, on proteins leading to denaturation, and on nuclear DNA leading to its cross linkage and DNA damage $[3,4,5]$. Oxidative stress produced by Cisplatin is the result of increased ROS production and inhibition of enzymes responsible for degradation of ROS which are superoxide dismutase, glutathione peroxidase, and catalase [4, 5].Inflammation was also found to play an important role inCisplatin cellular injury andnephrotoxicity $[3,4,5]$. In clinical practice blood urea, serum creatinine, serum albumin are the most important parameters for evaluation of renal damage $[3,4,5]$. Because, urea and creatinine are filtered by glomeruli and secreted in the urine, elevated levels of urea and creatinine were considered as an important parameter of impairment of renal function especially tubular and glomerular injury with 
reduction of glomerular filtration rate [3, 4, and 5]. Reduction in serum albumin is a sign of glomerular and tubular injury, because of leakage of large molecules like albumin into glomerular filtrate by injured glomeruli and inability of injured renal tubules especially proximal renal tubules to reabsorb leaked protein from glomerular filtrate [3, 4].

\section{Materials and Methods:}

Thirty-SixIraqi white domestic male rabbits were obtained from Baghdad animal market and housed in the animal house of Histology Department/AlMustansiriaCollege of Medicine. The animals aged 6 8 months and weighing 1.5-2.0 kg. Every three animals were house in one cage and have free access to water and food. The animal house was provided with proper ventilation and controlled temperature, and 12 hours cycle of dark and light. Before starting the experiment, rabbits were left for three days in animal houseto acclimatized to the new environment. The study was approved by the Ethical Committee of Al-Mustansiria College of Medicine. The study was conducted between February 14, 2016 - June 2, 2016 at Department of Pathology and Forensic Medicine, Al-Mustansiria College of Medicine. Preparation of Cisplatin:Cisplatin injections were obtained fromEbwePharma Austria and given as single intraperitoneal injections in a dose of $6.5 \mathrm{mg} / \mathrm{kg}$ body weight/24 hours through [7]. Experimental Design:Animals were randomly divided into three experimental groups each consists 12 rabbits as follows: Control group: Receives $3 \mathrm{ml}$, normal saline intraperitoneally daily in one injection. Cisplatin group: Receives Cisplatinintraperitoneallyin a dose of $6.5 \mathrm{mg} / \mathrm{kg}$ body weight/day in one injection for five days starting from $5^{\text {th }}$ day of experiment. Pomegranate group: Receives Cisplatindaily intraperitoneally $6.5 \mathrm{mg}$ $/ \mathrm{kg}$ body weight and pomegranate juiceorally in a dose of $30 \mathrm{gm} \mathrm{/} \mathrm{kg}$ body weight / day) in divided in three divided doses. Collection of blood: The blood was aspirated directly from the heart after animal wasfully anesthetizedusing techniques of blood aspiration from rabbits introduced by Medical Center Animal Care and use/Duke University [7, 8]. Rabbit Dissection: Dissectionstarted by placing animal on its back and clipping of its legs to the sides. Dissection started by opening the abdomen in the midline by scalpel from xiphisternum to the pelvis. Opening of the abdomen then completed by dissection of anteriorabdominal wall with a scissor. After opening abdominal cavity,the kidneys were taken after separation from its attachments. Then $1 / 2$ from each kidney is sliced at $2-3 \mathrm{~mm}$ thickness and left in $10 \%$ buffered formal - saline for histopathology. Second halves from both kidneys were freshly homogenized with a homogenizer and kept in deep freeze for MDA and GSH estimation by ELIZA. Investigations Parameters in the blood: Blood urea, Serumcreatinine, and Serum albumin by spectrophotometer using kits from Linear company. Serum tumor necrosis factor alpha (TNF - $\alpha$ ) level by ELIZA technique using ELIZA kit from Biocusa company. Parameters in kidney tissue homogenates: Kidney tissue MDA (Malodialdehyde), andKidney tissue GSH (Glutathione) levels using ELIZA kits from Biocusacompany. Histopathology Scoring of renal tubular injury; Sections from both kidneys were taken to determine the degree and score of renal tubular damage according to method by (Ramesh and Reeves 2004; Liu et al. 2006), [8, 9] table-1. Numbers of neutrophils in histopathological section is calculated by counting the number neutrophils per high power field after examining10 high power fields (x400), in outer stripe of medulla and taking the average number [5].

Table-1: Histopathological scoring of renal tubular damages.

\begin{tabular}{ll}
\hline Scores & Renal tubular necrosis \\
\hline 0 & None \\
\hline 1 & $<$ than $25 \%$ of tubules affected \\
\hline 2 & $25-50 \%$ of tubules affected \\
\hline 3 & $50-75 \%$ of tubules affected \\
\hline 4 & $>$ than $75 \%$ of tubules affected \\
\hline
\end{tabular}

Statistical Analysis: Statistical analysis was performed using IBM SPSS version 24 statistical software and Microsoft Excel 2010. Numerical data were obtained as mean and standard error. The $t$-tests and ANOVA tests were carried out for comparison between the control group, treated group and nontreated group [6]. The results were considered statistically significant when the ( $p$-value) is below 0.05 .

\section{Results:}

In control group the mean levels of blood urea, serum creatinine, and serum albumin were $35.34 \pm 1.40 \mathrm{mg} / \mathrm{dl}$, $0.54 \pm 0.06 \mathrm{mg} / \mathrm{dl}$, and $3.2 \pm 0.06 \mathrm{gm} / \mathrm{dl}$ respectively. In cisplatin group the mean levels of blood urea, serum creatinine, and serum albumin were 125.56 \pm 0.53 $\mathrm{mg} / \mathrm{dl}, 4.84 \pm 0.08$, and $0.98 \pm 0.01 \mathrm{gm} / \mathrm{dl}$ respectively. In pomegranate group the mean levels of blood urea, serum creatinine, and serum albumin were $37.3 \pm 0.82$ $\mathrm{mg} / \mathrm{dl}, \quad 0.69 \pm 0.09 \mathrm{mg} / \mathrm{dl}, \quad$ and $3.1 \pm 0.2 \mathrm{gm} / \mathrm{dl}$ respectively. All results of blood urea, serum creatinine and albumin were with $p$-value $<0.05$, figure-1. 


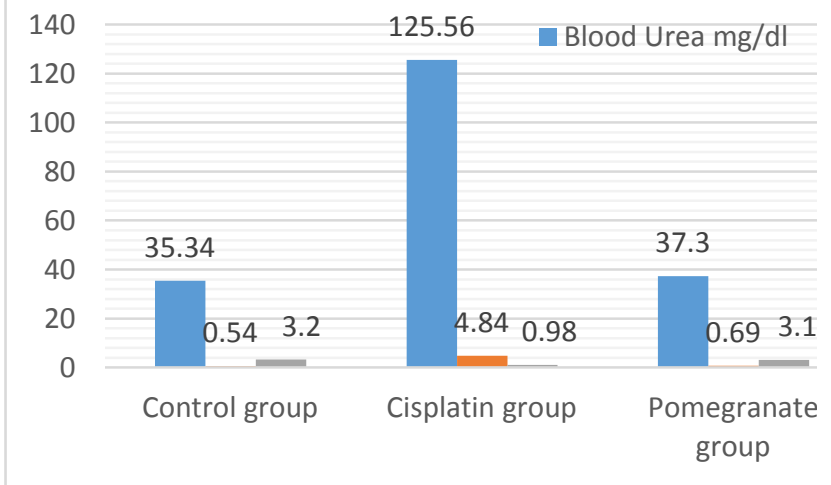

Figure-1: Mean of blood urea, serum creatinine, and serum albumin in study groups.

In control group the mean levels of serum TNF- $\alpha$, tissue MDA, and tissue GSH were $40.85 \pm 1.62 \mathrm{pg} / \mathrm{ml}$, $171.63 \pm 0.75 \mathrm{ng} \mathrm{MDA} / \mathrm{gm}$ of wet tissues, and $60.50 \pm 0.830$ nano-mol $/ g$ of wet tissues respectively. In cisplatin group the mean levels of TNF- $\alpha$ in serum, tissue MDA, and tissue GSH were $389.37 \pm 4.96 \mathrm{pg} / \mathrm{ml}$, $592.42 \pm 2.15 \mathrm{ng} \mathrm{MDA} / \mathrm{gm}$ of wet tissues, and $10.38 \pm 0.42$ nano-mol/g wet tissues wet respectively. While in pomegranate group the mean of serum levels of TNF- $\alpha$, tissue MDA, and tissue MDA were $43.13 \pm 1.69 \mathrm{pg} / \mathrm{ml}, 175.51 \pm 1.67 \mathrm{ng} \mathrm{MDA} / \mathrm{gm}$ of wet tissues, and $76.12 \pm 0.70$ nano-mol/g wet tissuesrespectively.All results with statistically significant with $\mathrm{p}$-vale $<0.05$, figure- 2 .

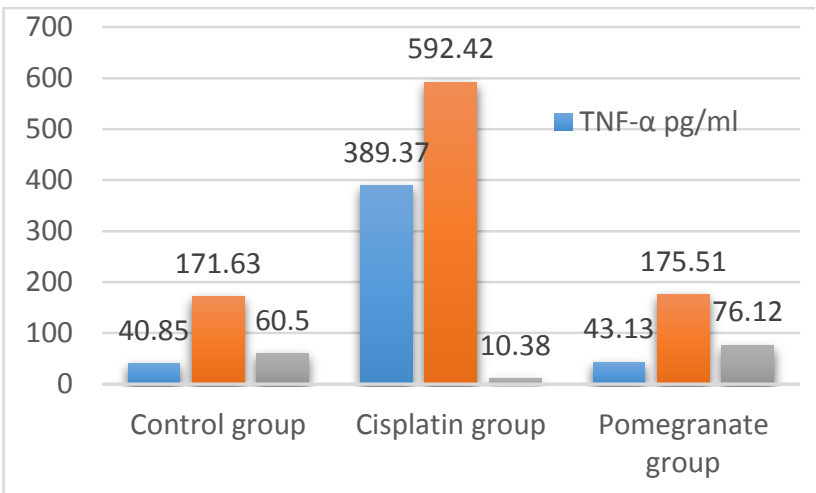

Figure-2: Mean values of serum TNF- $\alpha$, Tissues MDA and GSH levels.

Histopathological Examination:

HistopathologicalScores of renal tubular injuries and neutrophils in control group were zero, and in cisplatin group they were $3.49 \pm 0.12$, and $75.78 \pm 4.76$ respectively. While in pomegranate group they were $0.94 .51 \pm 20$, and $1.84 \pm 0.53$ respectively, figure-3.

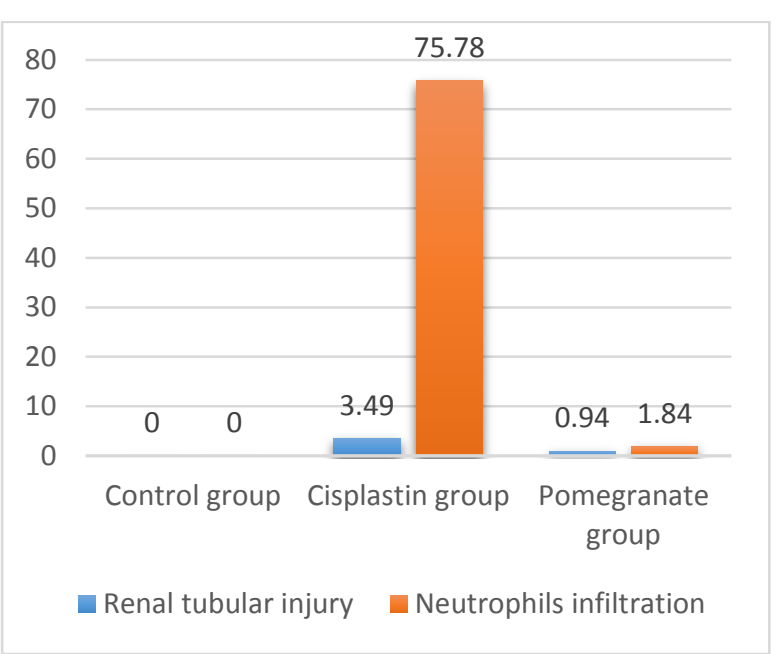

Figure-3: Mean score of renal tubular injury and neutrophils count in study groups.

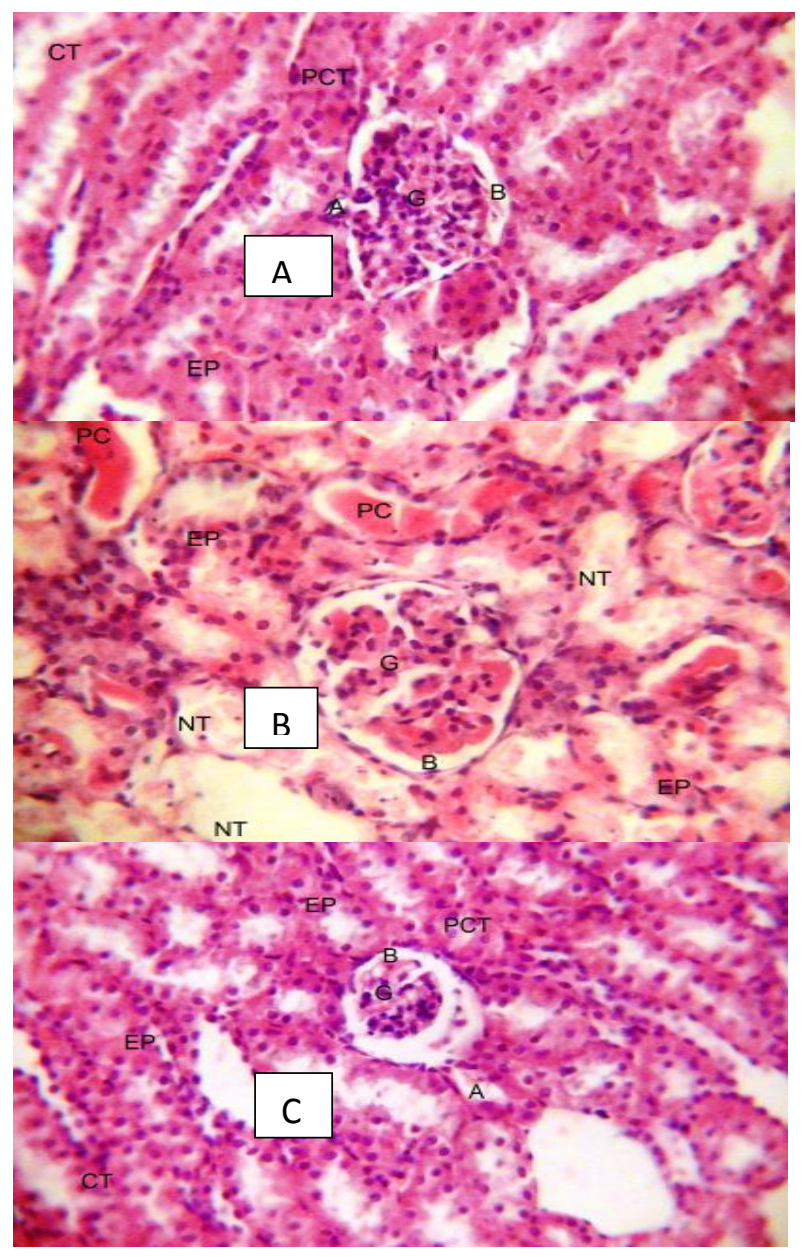

Figure-4: Kidney sections A- from control, B-from Cisplatin, and C-from Pomegranate group, H\&E X400). 


\section{Discussion:}

Cisplatin is a potent chemotherapeutic agent against most of solid organs cancer, but it has many side effects, the most important of which is nephrotoxicity $[3,4]$. The present study showed that sera from animals in the Cisplatin group had high blood urea and serum creatinine with low serum albumin levels compared to control group. These findings are signs of renal glomerular and tubular injury, and are evidences of Cisplatin nephrotoxicity. The Cisplatin-induced nephrotoxicity was also supported, in the present study, by histopathological findings of renal glomerular and tubular damage, and increased neutrophilic inflammatory cells in renal parenchyma of animals in Cisplatin group. It was also found that elevation in levels of blood urea and serum creatinine, and reduction in serum albumin correlated with the degree of renal injury. Because, cases with severe renal damage seen by histopathology in Cisplatin group had also high levels of blood urea, and serum creatinine and lower level of serum albumin and vice versa. Findings of present study support that Cisplatin in a nephrotoxic agent and high blood urea and serum creatinine with low serum albumin are important parameters of renal injury and deterioration of renal function. These agree with other studies by Seyed S B Mousavi et.al [3], AnjaWilmesaet al [4], Ferguson M A et al [5]. The TNF- $\alpha$, MDA, and GSH are markers for inflammatory reaction [3, 4]. Malonic acid dialdehyde (MDA) is an organic compound, and is a byproduct of lipid metabolism (lipid peroxidation) by especially by action of free radicals and reactive oxygen species (ROS) on membranes of cells $[3,4]$. Glutathione (GSH) is an antioxidant naturally present inside the cells of animals, and it is called "body's master antioxidant" [3]. The present study showed in Cisplatin group, there were elevations in serum levels of TNF- $\alpha$ by ELIZA, and elevation in levels of MDA, and lowering in levels of GSH in kidney tissue homogenate. Changes of these parameters pointing toward renal parenchymal damage by inflammation and oxidative stress [3, 4].Histopathological findings of same animal in Cisplatin group also showed severe renal glomerular and tubular damage with increased renal parenchymal neutrophilic infiltration. These biochemical changes were also correlated with histopathologicalfindings (the marked changes in these parameters were associated with severe histopathological changes, and vice versa). Findings from current study agree with results obtained by other studies such as, Ronald P. Miller et al [9], Pabla N et al [10], and Yuki Miyawaki et al [11]. The literatures reported that concomitant use of one of the natural anti-oxidants with Cisplatin reduces the renal glomerular and tubular injury [14, 15, 16, 17, 18], because the main cause for Cisplatin-induced nephrotoxicity is inflammation induced by ROS in oxidative stress. In past years, scientific researches on use of herbs in medicine found a great beneficial effect from the use of these natural products, such as thyme, pomegranate, Zofran, etc., in traditional ethnomedicine in many diseases in which the causes were inflammatory and oxidative stress [18, 19, 20, 21, 22]. The potent nephroprotective effect of pomegranate juice against Cisplatin-induced nephrotoxicity in the present study, has been shown by normal serum levels of blood urea, serum creatinine, albumin, and TNF- $\alpha$, and normal renal tissue levels of MDA and GSH in the rabbits of pomegranate group. Nephroprotective property of pomegranate has been also confirmed in present study by absence of renal parenchymal damage and/or inflammation in pomegranate group by histopathologic examination of renal tissues. The biochemical and histopathological findings agree with findings of other previous studies such as, MOHANA LAKSHMI et al [17], Aida Zarfeshany et al [18], Jurenka JS et al [19], Çimen KARASU et al [20], Jaya PreethiPeesa et al [21], ValérianBunel et al [22], and Abdullah Ozkok et al [23]. ConclusionsPure pomegranate juice in a dose of $30 \mathrm{gm} / \mathrm{kg} /$ day, orally), reduced cisplatininduced nephrotoxicity in rabbits and maintain normal renal function.

Authors' contributions: Dr. HussainAbadyAljebori contributed in planning of the study, dissection of rabbits,preparing of slides,histopathological examination of slides, editing and interpretation of results. Ali $\mathrm{H}$ Abady contributed in planning of the study preparation of rabbits, preparation of ciplatin and pomegranate juice injection of rabbits, and dissection of rabbits. Dr. JaffarNouriJaffaralalsaidissacontributed in histopathological examination of slides, editing, interpretation of results and statistical analysis of the results.

\section{References:}

1. Mark A Perazella, and Randy L Luciano. Review of select causes of drugs - induced acute kidney injury. Exper Rev ClinPharmacol.2015; 8 (5): $367-71$.

2. Seyed $S$ B Mousavi, and Zerrati A. Cisplatin Induced nephrotoxicity. J BirjandUni Med Sci2014; vol 20 (4): $327-37$.

3. Seyed S B Mousavi, and Zerrati A. Cisplatin Induced nephrotoxicity. J BirjandUni Med Sci 2014; vol 20 (4): $327-37$.

4. AnjaWilmesa, Chris Bielowb, Christina Ranningerc, Patricia Bellwond, Lydia Aschauera, and Alice Limonciela, et al. Mechanism of cisplatin proximal tubule toxicity revealed by integrating transcriptomics, proteomics, metabolomics and biokinetics. Toxicology in Vitro. December 2015; 30 (1):117-27.

5. Ferguson $M A$, Vaidya $V S$, and Bonventre $J V$. Biomarkers of nephrotoxic acute kidney injury. Toxicology2008; 245 (3): 182-93. 
6. IBM SPSS Statistics v24 Free download. Retrieved online at 25th of June 2017 from: https://rahimsoft.org/ibm-spss-statistics-v24-free-download/.

7. Turner Patricia, BrabbThea, Peko, Cynthia, and Vasbinder Mary Ann. administration of Substances to Laboratory Animals: Routes of Administration and Factors to Consider. Journal of the American Association for Laboratory Animal Science. September 2011; 50(5): $600-613$.

8. Duke Animal Care and Use Program. Guidelines and Suggestions. Retrieved online in January 2017 from: https://sites.duke.edu/oawa/policies-proceduresguidelines/guidelines-and-suggestions.

9. Ronald P. Miller, Raghu K. Tadagavadi, Ganesan Ramesh and William Brian Reeves. Mechanisms of Cisplatin Nephrotoxicity. Toxin 2010; 2(11): 2490518.

10. PablaN, and Dong Z. Cisplatin nephrotoxicity: Mechanisms and renoprotective strategies. Kidney International. May 2008;73(9):994-07.

11.Yuki Miyawaki, Masaaki Ueki, Masaaki Ueno, TakehikoAsaga, Masaaki Tokuda, and GotaroShirakami. D - Allose Ameliorates Cisplatin Induced Nephrotoxicity in Mice. Tohoku J Exp Med. 2012; 228: 215 - 21 .

12.Pei Ma, Sen Zhang, Xinlin Su, GuixingQiu, and Zhihong Wu. Protective effects of icariin on cisplatininduced acute renal injury in mice. Am J Transl Res. 2015; 7 (10):2105 - 4. ISSN:1943-8141/AJTR0014285. 13. IstvanAranya, and Robert L Safirstein. Cisplatin nephrotoxicity.Seminars in Nephrology. September 2003; 23(5): 460-64.

14. Kim M., Yang HN, Kim H., Jo S., Cho WY., and Kim HK: IL-10 mediates rosiglitaone-induced kidney protection in cisplatin nephrotoxicity. Journal of Korean Academy of Medical Sciences. 2010; 25(4):557-63.

15. Ali Karimi, MaedehMajlesi, and Mahmoud Rafieian-Kopaei. Herbal versus synthetic drugs; beliefs and facts. J Nephropharmacol. 2015; 4(1): 27 30.

16. ShabnamHajian, Mahmoud Rafieian-Kopaei, and Hamid Nasri. Renoprotective effects of antioxidants against cisplatin nephrotoxicity. J Nephropharmacol. 2014; 3(2): 39-42.

17.MOhana Lakshmi, SushaKiranReddyt, and Sandhya Rani.A REVIEW ON MEDICINAL PLANTS FOR NEPHROPROTECTIVE ACTIVITY. Asian Journal of Pharmaceutical and Clinical Research. 2012; 5 (4): 8 - 14.

18. Aida Zarfeshany, Sedigheh Asgary,1 and ShaghayeghHaghjooJavanmard. Potent health effects of pomegranate. Adv Biomed Res. 2014; 3(100). doi: 10.4103/2277-9175.129371.

19. Jurenka JS. Therapeutic applications of pomegranate (Punicagranatum L.): A review. Altern Med Rev. 2008; 13:128-44.

20. Çimen KARASU, Ahmet CUMAOĞLU, Ali Rifat GÜRPINAR, Murat KARTAL, Lucia KOVACIKOVA, and Ivana MILACKOVA. Aldose reductase inhibitory activity and antioxidant capacity of pomegranate extracts. InterdiscipToxicol. 2012; 5(1):15-20. doi: 10.2478/v10102-012-0003-8.

21. Jaya PreethiPeesa. Nephroprotective Potential of Herbal Medicines: A Review. Asian J Pharm Tech. 2013; 3(3): $115-18$.

22. ValérianBunel, Marie-Hélène Antoine, JoëlleNortier, Pierre Duez, and Caroline Stévigny. Potential nephroprotective effects of the Chinese herb Angelica sinensis against cisplatin tubule-toxicity. Pharmaceutical biology. 2015; 53 (7): 985 - 94.

23. Abdullah Ozkok and Charles L. Edelstein. Pathophysiology of Cisplatin-Induced Acute Kidney Injury. Journal of Med Research International. August 2014; (2014) Article ID 967826:17. doi.org/10.1155/2014/967826. 